\title{
Fetus Support Manipulator with Flexible Balloon-Based Stabilizer for Endoscopic Intrauterine Surgery
}

\author{
Hongen Liao ${ }^{1}$, Hirokazu Suzuki ${ }^{2}$, Kiyoshi Matsumiya ${ }^{2}$, Ken Masamune ${ }^{2}$, \\ Takeyoshi Dohi ${ }^{2}$, and Toshio $\mathrm{Chiba}^{3}$ \\ ${ }^{1}$ Graduate School of Engineering, the University of Tokyo \\ ${ }^{2}$ Graduate School of Information Science and Technology, the University of Tokyo \\ ${ }^{3}$ Department of Strategic Medicine, National Center for Child Health and Development \\ 7-3-1 Hongo, Bunkyo-ku, Tokyo 113-8656, Japan \\ liao@miki.pe.u-tokyo.ac.jp
}

\begin{abstract}
A novel manipulator is described for stabilizing fetus and preventing it from free-floating during the endoscopic intrauterine surgery. Minimally invasive endoscopic fetal surgery enables intrauterine intervention with reduced risk to the mother and fetus. We designed and fabricated a prototype of a fetus supporting manipulator equipped with a flexible bending/curving mechanisms and a soft balloon-based stabilizer. The flexible bending and curving mechanisms enable the stabilizer to reach the target sites within the uterus under an ultrasound-guidance. The balloon-based stabilizer could be inserted into the uterus with a small incision for entry. The accuracy evaluation showed that the maximum error of the curving mechanism was as small as $7 \mathrm{~mm}$ and the standard deviation of the bending mechanism was just $1.6^{\circ}$. In the experiments using a fetus model, the manipulator could be well controlled under ultrasound guidance and its curving mechanism with the balloon-based stabilizer could be clearly visualized during the implementation of fetus model supporting. The manipulator has the potential to be used in minimally invasive intrauterine surgery, though further improvements and experiments remain to be carried out.
\end{abstract}

\section{Background}

Myelomeningocele is one of the most common congenital defects of the central nervous system [1]. It is a neural tube defect with spinal bonny malformation leading to an incomplete spinal canal. This can result in the dorsal protrusion of the spinal cord and its covering membranes. Myelonmeningocele accounts for about $75 \%$ of all cases of spina bifida and may occur in 1 per 1000 infants.

Since the neural damaging process like the obstacle of cerebrospinal fluid circulation, irrigation and absorption start before birth irreversibly, current neonatal surgical repair does not aim to reverse the central nervous damages, but aims merely to preserve the residual nervous function. Previous studies showed that the intrauterine environment may cause secondary injury to the spinal cord that is already dysplastic [2]. This suggested that in utero repair of the myelomeningocele could minimize this secondary injury and improve the life-long neurologic outcome [3]. 
Surgical treatment of the myelomeningocele during 19-25 weeks of gestation is expected to improve the central/spinal cord nerve functions in utero preventing an association of hydrocephalus. The rationale of this procedure is based on the fact that the compliance of the fetal nervous system is high enough to be normalized in terms of its morphology due to the immature fetal myelin tissues not yet fully formed at this developmental stage. Furthermore, regeneration of damaged neural tissues is potentially better in earlier stages.

In recent years, endoscopic technology has provided a less invasive approach to surgical treatment. Minimally invasive endoscopic fetal surgery enables intrauterine intervention with reduced risks to the mother and fetus [4]. The problem is that the fetus is floating in the amniotic fluid and its movement will disturbe the surgical treatment. Then, it is necessary to develop a fetus-holding device to support the fetus within the uterus. In a previous research, we attempted to develop a stainless steel fetal stabilizer [5]. The outcome of this research was unfavorable although the fetus was fixed well. Other device was a suction type silicone tube stabilizer equipped with small holes to apply negative pressures on the fetal skin [6]. However, this device was likely to cause fetal tissue congestion during a long time surgery.

To overcome these issues, we propose a newly-developed manipulator with a flexible mechanism and a balloon-based supporting stabilizer. The outcome of mechanical performance tests and surgical practice tests using a fetal model under ultrasound guidance will be also described.

\section{Methods}

We have designed and fabricated a prototype of the fetus supporting manipulator, aimed at proposing a flexible and soft stabilizer for supporting the fetus in the endoscope intrauterine surgery. In this section, fetus supporting method, system requirements, configuration, and the apparatus of the prototype are described.

\subsection{Requirements of Fetal Surgery}

The gestational age selected for myelomeningocele treatment is at 19-25 weeks corresponding to the characteristics of the fetus. The fetus in the target gestation is very small with a length of about $30 \mathrm{~cm}$ and weight of about $500 \mathrm{~g}$. The volume of the amniotic fluid is about $500 \mathrm{ml}$.

The fetal surgery is different from the other forms of surgery as follows. First, the fetus is fragile; the surgical instrument needs to be made small and flexible enough from hurting the fetus and the placenta. Second, the insertion sites for the surgical instrument into the uterus depend on the position of the placenta and the umbilical cord. Third, the operation space for instrument within the uterus is limited and the quality of endoscope image falls off due to the cloudy amniotic fluid. Those conditions limit the design of fetal surgical instrument.

\subsection{Fetus Stabilizing Mechanism and the Stabilizing Procedure}

During the myelomeningocele surgical repair, the forces pushed or pulled to the fetus will change the position and the posture of the fetus. In order to prevent the fetus from 
moving or rotating, the points supported to the abdomen side corresponding to the back of the fetus are required. Furthermore, it is necessary to support the breast of the fetus since the head is heavy. Considering of above two requirements, the designed fetal stabilizer should be enable supporting the abdomen side of the fetus from breast to the abdomen softly.

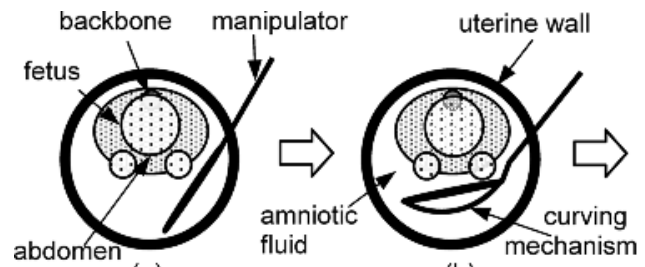

(a)

(b)

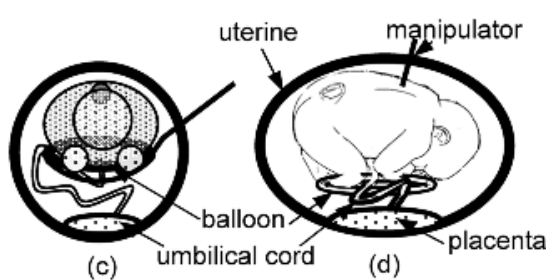

(d)

Fig. 1. Fetus supported by a balloon-based flexible manipulator. The insertion procedure shown in (a)-(c): (a) insert the manipulator straightly. (b) bend and adjust the curving mechanism to a optimal position. (c) pump the balloon by injecting physiological saline and stabilize the fetus. (d) viewed from left side.

To meet the requirements mentioned above, we developed a fetal stabilizing mechanism with a balloon that swells into a circle shape to support the fetus (Fig.1c,d). This balloon-based stabilizer is possible to support the fetus softly without any physical damage. We can fold the balloon and insert it into the uterus from a small opening hole. Furthermore, we use inject physiological saline from a syringe to swell the balloon. The injected amount and the pressure are controlled by PC. Even the balloon is broken during the operation it will not influence the fetus. The inserted part of the bending mechanism is covered by silicon so that the fetus and the placenta will not be injured.

The procedure of inserting the balloon-based stabilizer to the uterus and stabilizing the fetus with the ultrasound guidance is shown in Figure 1(a)-(c).

1) We insert the instrument with a shrunk balloon according to the position of the fetus. The spatial relationship of the fetus, the uterine wall and the instrument is observed using an ultrasonic diagnosis device.

2) The bending angle of the link part and the curving part of the manipulator are controlled by a PC according to position of the fetus. The bending and curving mechanisms are crooked and inserted into the required position with the guidance of intra-operative ultrasonic image.

3) When the silicon covered curving part arrive the abdomen of the fetus, we inject the physiological saline into the balloon and adjust the balloon to optimal position for stabilizing the fetus.

The circle-shape balloon supports the fetus near the hand and the foot. The umbilical cord passes by the balloon as shown in Fig.1c. 


\subsection{System Overview}

The prototype of the fetal support manipulator is comprised of three units: the flexible manipulator unit with a balloon-based stabilizer and a curving mechanism; the PC for controlling the bending mechanism and the syringe; the ultrasonic device for viewing the intra-operative situation of the fetus and the instrument (Fig.2).

The balloon is connected with a syringe. The pressure is generated by an electric linear slider with a syringe $(60 \mathrm{ml})$ similar to a syringe pump. The manipulator is held by the surgeon and controlled by the PC. The manipulator is performed under the guide of the ultrasonic image.

In general endoscopic surgery, since the viewing zone of endoscope is small and sometime the endoscope can not reach the narrow area, an ultrasonic image is used for assisting the endoscope surgery. The ultrasound is an ideal imaging modality for the diagnosis in obstetrics and gynecology. We use ultrasound to acquire the real-time intra-operative information. The position of the fetus and the umbilical cord could be identified when we insert the stabilizer into the uterus.

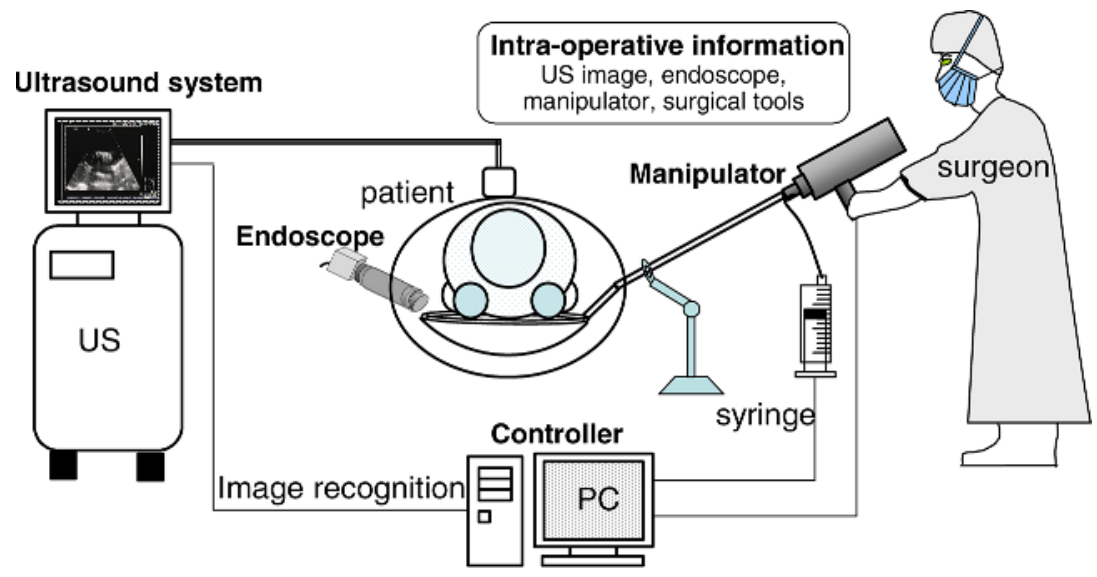

Fig. 2. Fetus support manipulator system for intrauterine surgery

\subsection{Mechanism of the Manipulator}

We put a tube inside the linkage for injecting the saline into the balloon (Fig.3c). The linkage mechanism made of stainless steel $6 \mathrm{~mm}$ in diameter. The length of the curving mechanism is determined by the size of balloon. Since the circumference of the fetus's body is about $15 \sim 20 \mathrm{~cm}$ and the diameter of the corresponding balloon is about $10 \mathrm{~cm}$, we developed a curving mechanism with $15 \mathrm{~cm}$ length which is suitable for enlarging the balloon.

In order to minimize the size of the insertion hole, we developed a curving mechanism that is comprised of a board spring and a wire-driven mechanism (Fig.3d). The balloon could be stored between the curving mechanism and the linkage part.

To the bending mechanism, we adopt a linkage-driven approach so that the manipulator enables a high stiffness (Fig.3b), durability and accurate performance than a wire-driven approach. Since the latex may trigger the fetus allergic reaction, 
they will not be considered as material for the supporting device. The balloon is made of polyethylene so that fetus could be supported almost without pressure. The area in contact with the fetus becomes larger and the pressure to the fetus becomes smaller when the balloon is made bigger. However, the incision to the patient would be large. As shown in Fig. 3c, the diameter of the curving mechanism is $6 \mathrm{~mm}$.

The tip part of the manipulator is sterilized by the autoclave (high-pressure steam), and connected with the actuator by draping mechanism.

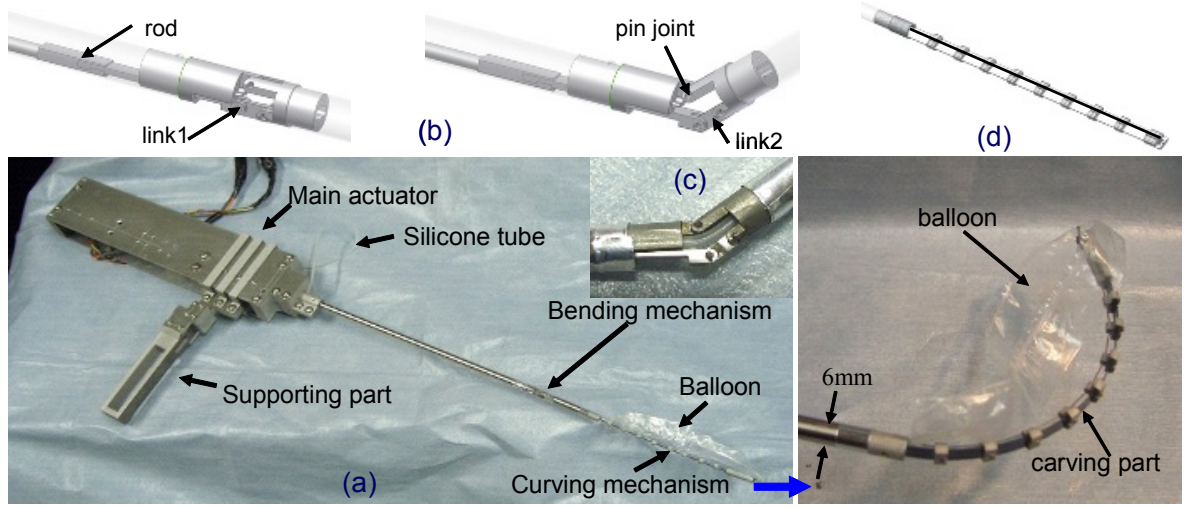

Fig. 3. Linkage bending mechanism, carving mechanism and balloon-based stabilizer

\section{Experimental Results}

The manipulator has two main functions: bending and curving the mechanism to the optimal position in the uterus; swelling the balloon to support the fetus. First, we evaluated the performance of the stabilizer curving and the linkage bending. Secondly, using the stabilizer to support the fetus, an experiment was carried our on a fetus model. We also evaluated the feasibility of the ultrasound guided manipulator.

\subsection{Accuracy Evaluation of Stabilizer Curving Mechanism}

We carried out experiment to evaluate the mechanical performance of the stabilizer bending mechanism. In order to swell the balloon to the required shape in fetus supporting, it is necessary to bend the curving mechanism and shorten the distance between the root and the tip part of the board spring. Furthermore, even the curving mechanism was not contacted the womb during the curving process, it is possible to touch the womb in recovering procedure if the hysteresis existed. The first set of tests aimed to measure the distance change from the root point to the tip end point of the curving linkage. We use a camera (QV-R51, CANON) to capture the pose of the curving process and measure the position of the tip end point. It was found that the hysteresis was existed and the error was $7 \mathrm{~mm}$ in maximum (Fig.4). This result showed the accuracy of curving mechanism is sufficient in stabilizing the fetus. 


\subsection{Accuracy Evaluation of Linkage Bending Performance}

The second set of tests assessed the performance of the linkage bending mechanism. We inserted and adjusted the stabilizer corresponding to the position of the placenta using the bending mechanism. It is necessary to ensure the bending range of the linkage mechanism. We evaluated the accuracy of the bending angle from $-45^{\circ}$ to $45^{\circ}$ and compared the results with the theoretical values. It was found that the measured values were satisfied with the theoretical value (Fig.5). The maximum error of the bending angle was $5^{\circ}$ and the standard deviation was $1.6^{\circ}$.

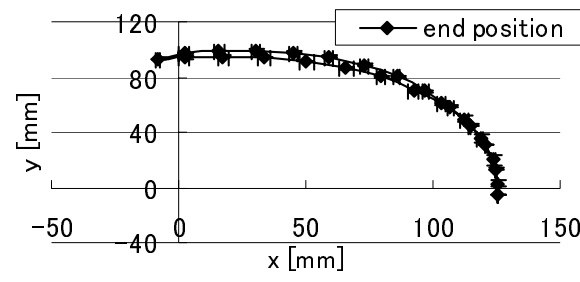

Fig. 4. Tip end point positions of the manipulator

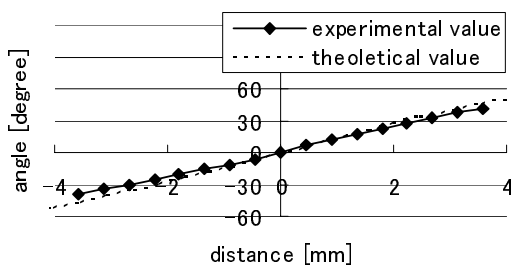

Fig. 5. Relationship between the translation distance and the bending angle

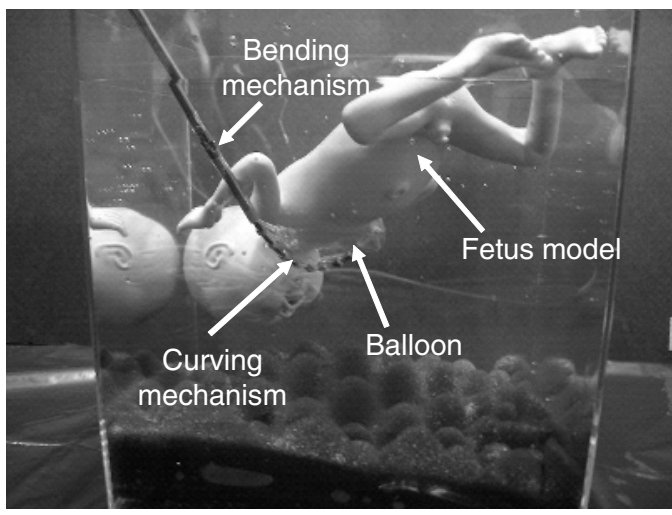

Fig. 6. Manipulator stabilizes the fetus model softly

\subsection{Fetus Supporting Experiment Using Fetus Model}

The flexible fetal supporting manipulator was applied to a fetus model stabilizing experiment. The fetus model weighs $600 \mathrm{~g}$ with volume of about $600 \mathrm{~cm}^{3}$ and length of $30 \mathrm{~cm}$. The long radius of the abdomen is about $6 \mathrm{~cm}$. The fetus model was suspended in a water tank. We inserted the manipulator from the side of the fetus, bending the linkage mechanism and crooking the stabilizing mechanism. The balloon was swelled and adjusted to support the fetus from the abdomen side. Figure 6 showed that the developed manipulator could support the fetus model and stabilize the posture. The picture also showed that only the balloon contacted with the fetus and the metal curving mechanism was not in contact with the fetus. The balloon could 
stabilize the fetus and prevent it from moving or rotating. The experiment showed that the fetus model could be supported and lifted up sufficiently using the manipulator.

\subsection{Ultrasound Guided Manipulator Insertion}

We carried out experiments to evaluate the feasibility of the ultrasonic image guidance to the insertion of the manipulator. The situation of the fetus model in the action of the manipulator is also identified using the ultrasonic device.

The first set of tests is to evaluate the ultrasonic image of the manipulator without placing the fetus model. Figure 7 a showed the situation when the balloon was shrunk. The curving mechanism was observed clearly. Figure $7 \mathrm{~b}$ showed that both of the mechanism and the balloon were observed clearly after the balloon was swollen up.

The second set of tests is to use fetus model to similar an implementation of fetal surgery with the developed manipulator. Figure 7c illustrated that the fetus model and the manipulator could be observed. We adjusted the stabilizer to the optimal position to support the fetus (Fig.7d). The manipulator and the curving mechanism could be observed under the fetus model clearly.

(a)
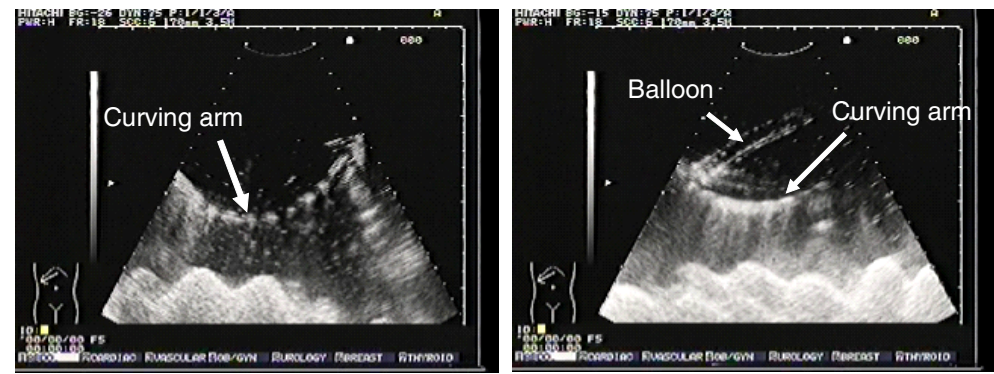

(b)

(c)
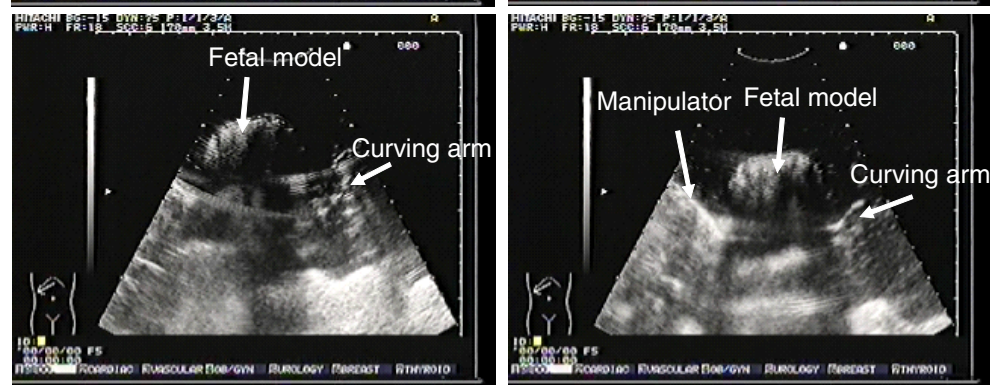

(d)

Fig. 7. Ultrasound guided manipulator insertion in stabilizing the fetus model (a) the curving mechanism was observed clearly. (b) both of the curving mechanism and the swollen balloon were observed. (c) the fetus model and the manipulator is observed . (d) The manipulator and the curving mechanism could be observed under the fetus model.

\section{Discussions and Conclusion}

The prototype manipulator has the potential to be successfully inserted into the uterus during the endoscope intrauterine surgery, while the balloon-based stabilizer has the 
potential to support the fetus softly. The experimental results demonstrated feasibility of the developed manipulator. First, the hysteresis of the curving mechanism showed as small as a 7-mm error and the accuracy of the linkage bending mechanism revealed the standard deviation of bending angle was just $1.6^{\circ}$. Second, the fetus model could be supported and lifted up by the balloon based stabilizer. Third, the manipulator could be controlled and the curving mechanism with balloon in the stabilizer could be clearly observed under ultrasound guidance.

In the minimally invasive fetoscopic surgery, the balloon is expected to be larger for fetus stabilization unlike its insertion sites which should be made as small as possible. Then, it is necessary to improve the method of balloon folding and storing.

The use of ultrasound was not enough to give information below the model (Fig. 7c), although it clearly visualized the manipulator and the fetus model as well. To achieve a full intra-operative guidance, the endoscope could be also available.

In conclusion, we reported a newly developed method of supporting the fetus during the endoscope intrauterine surgery. The flexible manipulator could be controlled to reach the optimal position in the uterus and the balloon-based device could stabilize the fetus softly. The manipulator is potentially helpful for minimally invasive intrauterine surgery, although further improvements and experiments are required.

\section{Acknowledgment}

This study was supported in part by the Grant-in-Aid of the Ministry of Education, Culture, Sport, Science and Technology in Japan, and Grant-in-Aid of the Ministry of Health, Labour and Welfare in Japan.

\section{References}

[1] D.C. McCullough, D.L. Johnson, "Myelomeningocele repair: technical considerations and complications," Concepts Pediat Neurosurg, Vol.8, pp29-40, 1988.

[2] D.S. Heffez, J. Aryanpur, G.M. Hutchins, J. Freeman, "The paralysis associated with myslomeningocele: Clinical and experimental data implicating a preventable spinal cord injury," Neurosurgery Vol. 26, pp.987-992, 1990.

[3] O.S. Aaronson, et al. "Robot-assisted endoscopic intrauterine myelomeningocele repair: A feasibility study," Pediatric Neurosurgery, Vol.36 No.2, pp.85-89, 2002.

[4] K.C. Oberg, A.E. Robles, et al. "Endocsopic intrauterine surgery in primates," Surgical Endoscopy, Vol.13, pp420-426, 1999.

[5] M. Dinh, J. Bruner, "Fetal Stabilizer for Intrauterine Surgery", Report of Vanderbilt University.

[6] Tsubouchi et al. "Development of the drawn-in type stabilizer for a fetus operation," The 14th conference of Japan computer-aided-surgery society, pp.45-46, 2005. 